\title{
Design and Evaluation of a High-Speed Aeroacoustic Wind Tunnel
}

\author{
Frank Giardino*1 and Joana Rocha ${ }^{\dagger 1}$ \\ ${ }^{1}$ Department of Mechanical and Aerospace Engineering, Carleton University, Ottawa, Canada, K1S 5B6
}

\section{Introduction}

During cruise flight, the main source of acoustic noise radiation within the aircraft cabin occurs as a result of structural vibrations of the flexible fuselage panels due to the random pressure fluctuation field imposed by the Turbulent Boundary Layer (TBL) [1]. Conventional methods of describing these interactions have relied on the use of various statistical semiempirical models which predict the behavior of the TBL pressure fluctuation spectrum. Each model however makes fairly different predictions with regards to the spectrum in the various frequency regions. There is therefore a need to not only assess these inconsistencies with experimental methods, but also to clarify the fundamental physical relationship between the turbulent structures and the resulting fluctuation signature. The present phase of this research initiative at Carleton University involves the design and fabrication of the new HighSpeed Aeroacoustic Wind Tunnel (HSAWT), which is capable of studying wall pressure fluctuation behavior at subsonic flow speeds up to typical cruise flight conditions.

\section{Wind Tunnel Design}

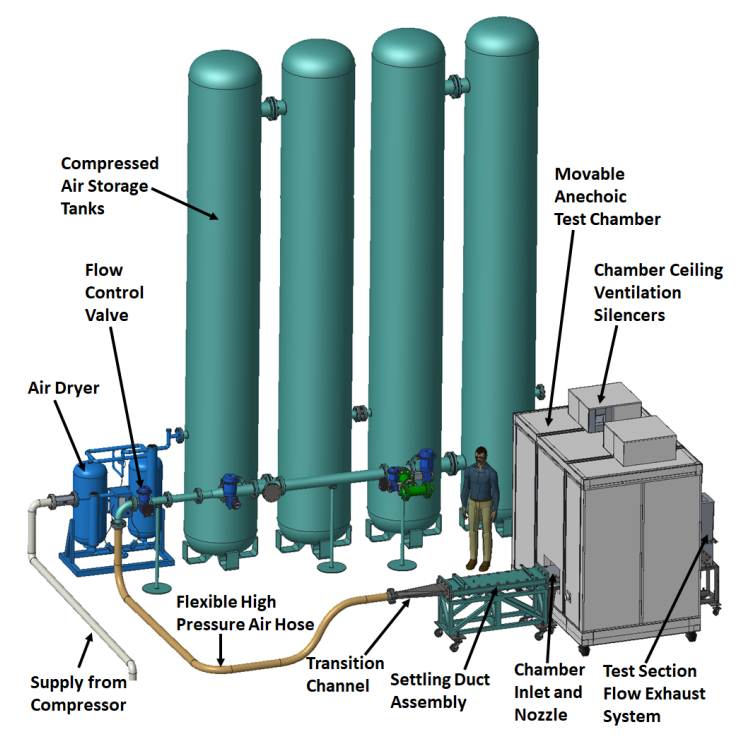

Figure 1: Carleton HSAWT facility.

The complete HSAWT facility is displayed in Fig.1, which makes use of a fully anechoic chamber to house an open jet test section. The wind tunnel is a blowdown type facility which operates using large compressed air reservoir tanks. Flow through the wind tunnel is achieved by discharging the air in the tanks in a controlled manner through a con-

\footnotetext{
*frankgiardino08@gmail.com

†Joana.Rocha@carleton.ca
}

trol valve. Constant test section flow conditions are achieved using a feedback control system which utilizes measurements of total and static pressure inside the test section to control the Mach number. Flow through the control valve is delivered to the chamber through a set of upstream components and flow straightening devices which are designed to properly guide and condition the flow. The flow is then accelerated into the test section to the required speeds using a nozzle assembly which is fed into the chamber and houses a 2D planar contraction profile. The chamber is built with acoustic paneled walls and is internally lined with anechoic wedges, providing the chamber with a cut-off frequency of $250 \mathrm{~Hz}$. Two ventilation holes on the chamber ceiling, which are fitted with acoustic silencers, allow for pressure equalization within the chamber at high speeds. The test section houses a test panel with an array of holes to flush mount pressure fluctuation sensors within the potential core of the jet. Flow over the panel is evacuated out of the chamber using a specially designed exhaust system. This system first contains a test section jet collector to properly capture the flow. The flow is then exhausted out of the chamber vertically through a set of diffuser duct components, as well as a $90^{\circ}$ corner fitted with turning vanes. The walls of the exhaust system are also acoustically treated to help suppress noise transmission back into the chamber.

\section{Wind Tunnel Characterization}

\subsection{Flow Control Response}

The flow control system was evaluated to determine the amount of steady run time available before the reservoir pressure is depleted. The freestream Mach number from the nozzle was computed along the centerline axis using pressure measurements from a suspended pitot-static probe. The results from this study indicate that the target Mach number is achieved within a settling time of roughly 12-15 seconds for all flow speeds. Constant control of the Mach number for at least 30 seconds is also reasonably achieved in the range from Mach 0.1 to 0.7. As the speed is increased to the maximum limit of Mach 0.8 however, the control system response is less steady, where the control valve seems to have a difficult time in compensating for the drop in tank pressure at a flow rate of approximately $3 \mathrm{~kg} / \mathrm{s}$. It is envisioned that future work in control system parameter optimization, combined with slight set-up modifications to lower the required mass flow rate, will provide improved control at higher speeds.

\subsection{Background Noise Levels}

Background noise levels within the test chamber were characterized to ensure that the expected magnitudes of the measured pressure fluctuation spectra are sufficiently large to 
avoid any signal contamination. Free-field chamber noise measurements were conducted using a Bruel and Kjaer 4944A 1/4" microphone, with the sensor placed perpendicular to the nozzle orifice at a horizontal distance of $1.2 \mathrm{~m}$. The results of this study confirmed that the chamber achieves an acceptable signal-to-noise ratio of at least $10 \mathrm{~dB}$ for almost all test conditions. Issues of signal noise contamination however may be possible at flow speeds around Mach 0.1 due to low levels of expected TBL surface pressure fluctuation energy. For this reason, alternative signal processing methods were implemented to ensure that noise contamination is minimized at all test section speeds.

\section{Surface Pressure Fluctuation Measurements}

Measurements of the surface pressure fluctuation spectrum were conducted using flush mounted microphones with a 5 $\mathrm{mm}$ pinhole cap above the sensor diaphragm for increased spatial and temporal resolution. A single sensor was used to measure the fluctuations at the point of interest while a secondary sensor was used as a reference signal in a noise cancellation technique. Fig. 2 contains results of the measured spectrum for the entire range of governable test section Mach numbers. The magnitude of the spectrum is represented on the decibel scale relative to $20 \mu P a$.

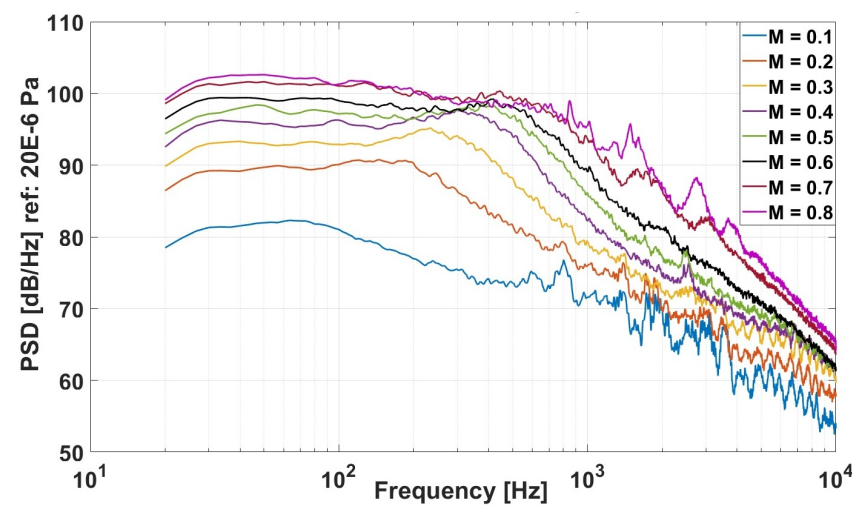

Figure 2: Comparison of measured surface pressure fluctuation spectra for various test section flow speeds.

The measured spectral behavior displays the expected trend of increasing power for higher flow speeds at all frequencies. The peaks of the spectra occur around $350 \mathrm{~Hz}$ and exhibit a main high frequency attenuation region of relatively constant slope. The spectra between Mach 0.2-0.6 also exhibit similar spectral peak and roll-off behavior as described by Rackl et al. [2] in their flight test measurements (slight "hump" in spectral peak before attenuation). In general, the spectra seem to display a lack of energy in the overlap/high frequency regions with a low spectral peak roll-off as the speed is increased to Mach numbers above 0.6.

In addition to the stand-alone measurements presented, the spectra were also compared with established spectrum models in literature as summarized by $[1,2]$. Fig.3 contain plots of the data comparisons with the model predictions at both the low and high test section velocity limits.

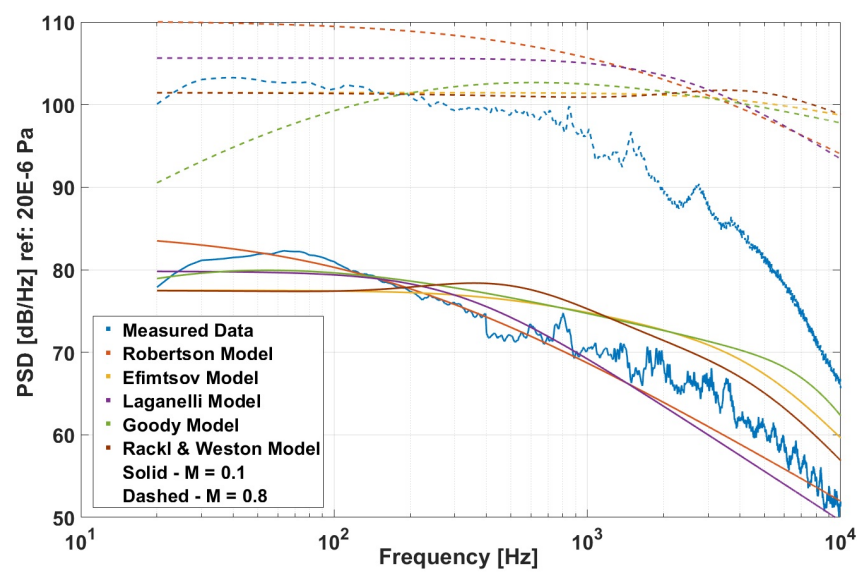

Figure 3: Comparison of measured surface pressure fluctuation spectra with predictions from literature models.

In general, the low speed data displays agreement with Robertson's model within the low frequency range, as well as a similar high frequency attenuation slope. For higher Mach numbers, the models predict a large energy shift in the spectrum to higher frequencies, thus the spectra seem to shift towards slightly lower energy levels predicted by Efimstov and Rackl \& Weston within the low frequency region. The high frequency regions however are still underrepresented in the data. This discrepancy in quick attenuation could be attributed to the sensor configuration utilized, underdeveloped TBL in the test section, and/or over estimated high frequency energy levels by the models for thinner TBL conditions.

\section{Conclusions}

The HSAWT facility commissioning and results of the initial pressure fluctuation studies conducted provides the necessary ground work for future research and improvements. The wind tunnel was able to obtain governed test section flow speeds up to those experienced in cruise flight with adequate low background noise levels. The implementation of the HSAWT seeks to provide an aeroacoustic test facility that is unique among many around the world, with the ultimate goal of mitigating the need for expensive flight tests to research TBL pressure fluctuations and their impact on the design of fuselage panels for the reduction of cabin noise generation.

\section{Acknowledgments}

The work presented was performed with the generous financial support from the Consortium for Research and Innovation in Aerospace in Quebec (CRIAQ) and the National Sciences and Engineering Research Council (NSERC).

\section{References}

[1] T.S. Miller, J.M. Gallman, and M.J. Moeller. Review of turbulent boundary-layer models for acoustic analysis. Journal of Aircraft, 49:1739-1754, 2012.

[2] R. Rackl and A. Weston. Modeling of turbulent boundary layer surface pressure fluctuation auto and cross spectra - verification and adjustments based on tu-14411 data. NASA Langley Research Center, Contractor Report CR-2005-213938:1-88, 2005. 